\title{
Uber das System der Verwandtschaftsnamen im Samojedischen
}

1. Seit langem wissen wir, dass fast jedes Detail der Wirklichkeit, also auch der gesellschaftlichen Wirklichkeit, mit sprachlichen Mitteln ausdrückbar ist. Das geschieht natürlich hier genauer, dort ungenauer. Die Tatsachen der Wirklichkeit ordnen sich nach irgendeinem System, und so schaffen auch die sprachlichen Mittel ein System. Besonders auffallend ist die Tatsache der Gesetzmässigkeit bei einer der ältesten gesellschaftlichen Formationen, im Falle nämlich der Verwandtschaft. Im Hinblick darauf aber, dass kein direkter Zusammenhang besteht zwischen den Tatsachen der Sprache und der Gesellschaft, ist Vorsicht geboten, wenn man von den sprachlichen Gegebenheiten auf das gesellschaftliche System schliessen will. In der Sprache werden sehr häufig solche Angaben konserviert, die aufgrund eines früheren gesellschaftlichen Faktors entstanden sind, welcher Faktor aber inzwischen durch einen neueren abgelöst wurde. Wir begegnen also ohne weiteres solchen nebeneinander existierenden sprachlichen Angaben, die jeweils einer anderen gesellschaftlichen Wirklichkeit entstammen.

Die Verwandtschaftsnamen bilden speziell ein solches System, für das die rein sprachwissenschaftliche Analyse nicht ausreicht; mit ständiger Aufmerksamkeit müssen jene Informationen verfolgt werden, die durch die gesellschaftliche Hierarchie vermittelt werden. Mit anderen Worten: die diesbezügliche Forschung muss sich einer soziolinguistischen Methode befleissigen.

2. Über die Untersuchung der Verwandtschaftsnamen existiert eine umfangreiche Literatur. Diese bezieht sich leider nicht auf die uralischen Sprachen, obwohl auch darüber bereits 
einige beachtenswerte Arbeiten erschienen sind. Doch wissen wir mit Ausnahme des Ungarischen, Finnischen und Estnischen in dieser Hinsicht ausserordentlich wenig über die anderen finnisch-ugrischen Sprachen. Und auch im Falle der erwähnten drei "grossen" Sprachen ist nicht jedes Detail geklärt. Die anfänglichen Versuche gingen i.a. nicht über die einfache Worterklärung hinaus, wobei man auf eine Reihe von unüberwindlich scheinenden Schwierigkeiten stiess. Und das nicht zufällig. Die Hierarchie der meisten finnisch-ugrischen Völker unterscheidet sich völlig von den westeuropäischen Systemen, so dass man bei einer Deutung der die verwandtschaftlichen Beziehungen ausdrückenden Termini den (gesellschaftlichen) Hintergrund nicht unberücksichtigt lassen kann. Die diesbezügliche Arbeit von Wolfgang Steinitz über die Ostjaken bedient sich schon fast vollständig soziolinguistischer Methoden und bedeutete somit eine wesentliche Neuerung verglichen mit den früheren Untersuchungen.

In einer besonders vernachlässigten Situation befinden sich die samojedischen Sprachen. Die Ursache kann auch darin liegen, dass wir im Falle der meisten samojedischen Sprachen nicht über die notwendigen Angaben verfügen; andererseits befanden sich die samojedischen Sprachen lange Zeit an der Peripherie des wissenschaftlichen Interesses.

In meiner Arbeit gehe ich nur auf die nenzischen und selkupischen verwandtschaftlichen Beziehungen ein. In Anbetracht dessen, dass das Nenzische der wesentlichste Vertreter des nördlichen und das Selkupische des südlichen Samojedentums ist, können wir mit Hilfe der daraus gewonnenen Ergebnisse den allgemeinen samojedischen Verhältnissen näher kommen. Das Bild wird natürlich nur dann vollständig, wenn sich die verwandtschaftliche Terminologie sämtlicher samojedischen Sprache klärt.

3. Diese Völker lebten bei Beginn des 20. Jh. in unerhört zurückgebliebenen gesellschaftlichen Verhältnissen. Nur allmählich machten sich die durch die Oktoberrevolution herbeigeführten Veränderungen geltend. Die Wurzeln der uralten Zustände reichten sehr tief; eine Änderung von einem 'Tag zum andern war nicht vorstellbar. Bei den Nenzen blieb zum 
Beispiel die alte Gentilordnung derart fest erhalten, dass auch die Kolchosen nur darauf aufbauend organisiert werden konnten.

Die Gentilhierarchie spiegelt sich auch in der Verwendung der Verwandtschaftsnamen wider. Unter Gens bzw. Sippe bzw. Geschlecht versteht man nach der ethnologischen Definition die auf unilateraler Abstammung gründenden verwandtschaftlichen Gruppen, deren Mitglieder jeweils durch das Wissen um die Abstammung vom gemeinsamen Vorfahren aneinander gebunden sind. Nach den Gentes wurden exogame Phratrien geschaffen, d.h. eine Eheschliessung war nur möglich, wenn der Partner zu einer anderen Gens gehörte. Die Gentilhierarchie hatte zur Folge, dass die Frau bei den Nenzen und Selkupen gleichermassen eine untergeordnete Rolle spielte, d.h. nur der Mann konnte ein Recht haben (wie z.B. bei der Erbschaft). Früher war auch die Monogamie nicht bindend, ein Mann konnte mehrere Ehefrauen haben, abhängig natürlich von seinem Besitztum bzw. Vermögen.

Die Auflösung der Gentilordnung begann bei den Selkupen früher, schätzungsweise im 18 . Jh. Dies prägte übrigens auch die Entwicklung der verwandtschaftlichen Terminologie.

4. Wie aus den späteren Ausführungen ersichtlich wird, ist die etymologische Zusammengehörigkeit oder Nichtzusammengehörigkeit unserer Belege aus den beiden Sprachen von unserem Standpunkt aus völlig gleichgültig. Natürlich haben wir es mit einer stattlichen Anzahl von Wörtern gemeinsamer Herkunft zu tun, schliesslich handelt es sich um relativ nah verwandte Sprachen. Diese Tatsache an sich verrät aber noch nichts über das heutige System der betreffenden Sprache, denn der unterschiedliche gesellschaftliche Hintergrund kann ja in der Bedeutung und Funktion der Wörter Veränderungen herbeiführen. Die Abweichungen in der Verwendung von Wörtern mit etymologisch identischer Bedeutung sind fast auf den ersten Blick augenfällig. Daraus folgt ferner, dass ich die ursprüngliche Bedeutung des verwendeten Terminus unberücksichtigt liess und - soweit dies nur vom Wortmaterial her möglich war - von der heutigen Verwendung des Wortes ausging. 
Über die verwandtschaftlichen Beziehungen der finnischugrischen Sprachen äusserte sich bereits Ahlqvist dahingehend, dass »das Familienleben bei den finnisch-ugrischen Völkern in eigener Weise entwickelt ist». $\mathrm{Zu}$ dieser eigenen Entwicklung gehört auch, dass die Verwendung solcher Benennungen häufig ist, die sich auf ausserordentlich viele, dem Anschein nach ganz unzusammenhängende Bedeutungskreise beziehen. Dieser Bedeutungsreichtum der Wörter kann trotzdem nicht zu einer Sinnesverwirrung führen, denn der Satzzusammenhang und die Situation machen die Bedeutung eindeutig, d.h. die Bedeutung des diesbezüglichen Terminus hängt davon ab, wer ihn benutzt, wer damit angeredet wird, von wem gesprochen wird usw. Diese Erscheinung ist übrigens auch dem Ungarischen nicht fremd.

Zum Beispiel:

nenz. jîri : Grossvater des Vaters

Grossvater der Mutter

Urgrossvater (Vater des Grossvaters;

Vater der Grossmutter)

Onkel (älterer Bruder des Vaters;

älterer Bruder der Mutter)

Schwager (älterer Bruder des Mannes)

Schwiegervater (Vater des Mannes) usw.

selkup. ilča Grossvater des Vaters

Grossvater der Mutter

Onkel (älterer Bruder des Vaters;

älterer Bruder der Mutter)

In diesem Fall ist es sehr wichtig, dass die oben gegebenen Bedeutungen der Termini vollständig gleichrangig sind, sie bezeichnen also nicht primär eine bestimmte verwandtschaftliche Beziehung. Die beiden Beispiele oben gehören zu den sog. klassifizierenden Termini, im Gegensatz zu denen, die sich stets auf eine einzelne konkrete Person beziehen; man pflegt diese auch bezeichnende Termini zu nennen. Z.B.: nenz. nebe 'Mutter'; selkup. emi 'Mutter'.

5. Zuerst wollen wir uns mit dem Verwandtschaftssystem 
im Nenzischen beschäftigen. ${ }^{1}$ Als Grundlage dient uns das Tundranenzisch. Die Transkription der Wörter sucht der phonematischen Lautschrift zu folgen.

5.1. Die Verwandtschaftstermini für die direkte Abstammung beziehen sich auf sechs Generationen, mehr oder minder genau. Darin ist natürlich der jeweilige Ausgangspunkt nicht enthalten, das EGo, auf das sich die Verwandtschaftsbeziehungen richten.

Mit Ausnahme der ersten Stufe der aufsteigenden Linie sind sämtliche Benennungen umfassende, klassifizierende Termini.

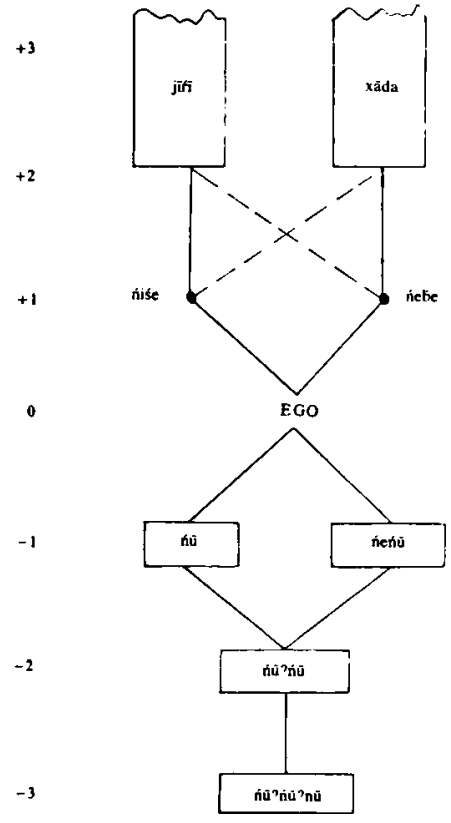

Abb. 1

${ }^{I}$ Als Quellen wurden die folgenden Werke verwendet: T. Lehtisalo, Juraksamojedisches Wörterbuch. Helsinki 1956; N. M. Tereščenko, Nenecko-russkij slovaŕ. Moskva 1965; Péter Hajdú, Chrestomathia Samoiedica. Budapest 1968; Kuprijanova, Terminologija rodstva... (Ǔc. zap. Ped. Inst. im. Gercena 101.). 
Die Evidenzhaltung von Vater und Mutter war für Ego in jeder Hinsicht wichtig; die Verwendung des unterscheidenden, bezeichnenden Terminus ist somit vollständig motiviert.

Die Bezeichnungen der zweiten und dritten Stufe der aufsteigenden Linie drücken bereits nur den vom EGo gerechneten Altersunterschied und das Geschlecht aus, unabhängig davon, ob es sich um Verwandtschaft väterlicher- oder mütterlicherseits handelt. Häufig handelt es sich also bei der mit jīri bezeichneten Person - in der Hierarchie der direkten Abstammung - um einen männlichen Verwandten von Eqo, der zwei oder mehr Generationen älter ist als EGo.

Auf der ersten Stufe der absteigenden Linie drücken $n \bar{u}$ und ṅeñu genau den geschlechtlichen Unterschied aus, während der Altersunterschied zu EGo nur global zum Ausdruck kommt und der relative Altersunterschied zwischen mehreren $n \dot{n}$ (oder néńu ) schon gar nicht.

Das Wort $n \bar{u}$ verfügt noch über eine zweite, nicht weniger wichtige Bedeutung: 'Kind'. Aufgrund der gesellschaftlichen Verhältnisse der Nenzen kann man leicht verstehen, dass in der Familie nur der Sohn wirklich als Kind galt, denn nur er war erbberechtigt, nur er konnte das Gentilwesen weiterführen. So entstand das auf ein Mädchen bezogene Wort ńeńū, d.h. 'Fraukind'. (Eine derartige Benennung der Kinder ist durchaus keine nenzische Besonderheit. Auch im Ungarischen sagt man recht häufig Nekem lét gyerekem van 'Ich habe zwei Kinder', wenn man damit ausdrücken will, dass es sich "selbstverständlich» um Söhne handelt. Handelt es sich aber nicht um Söhne, kann man oft die folgende Ausdrucksweise vernehmen: Nekem $c s$ a $k$ kít lányom van 'Ich habe nur zwei Töchter'.)

Aller Wahrscheinlichkeit nach ist das Wort ńn also älter als ńeńu und die ursprüngliche Bedeutung war 'Kind'. Das auf das weibliche Geschlecht hinweisende Element ne ist erst später an das Grundwort getreten und kann in seiner heutigen Funktion als Präfix betrachtet werden.

Die weiteren Stufen der absteigenden Linie differenzieren schon nicht mehr nach dem Geschlecht. Grammatisch ist $\dot{n}^{\prime} \vec{u} \hat{n}^{\prime} \bar{u}$ eigentlich eine Possessivkonstruktion 'das Kind des Kindes' und theoretisch kann diese Konstruktionsart auch auf der 
unteren Stufe weiterentwickelt werden, was praktisch jedoch kaum geschieht.

5.2. Das durchschaubarste System der Seitenlinien-Verwandtschaft bezieht sich auf die von ein und denselben Eltern abstammenden Geschwister. Die Termini unterscheiden nach dem Geschlecht, und auf zwei Stufen auch nach dem Alter. Ferner gibt es auch solche Benennungen, wo entweder das eine oder das andere Moment in den Vordergrund tritt: im

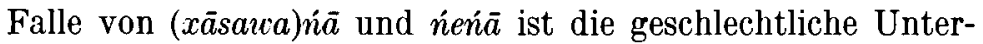
scheidung wichtig, das Alter wird irrelevant, während bei ṕebe das Genuszeichen irrelevant ist und das geringere Alter denn bei EGo den relevanten Faktor bildet.

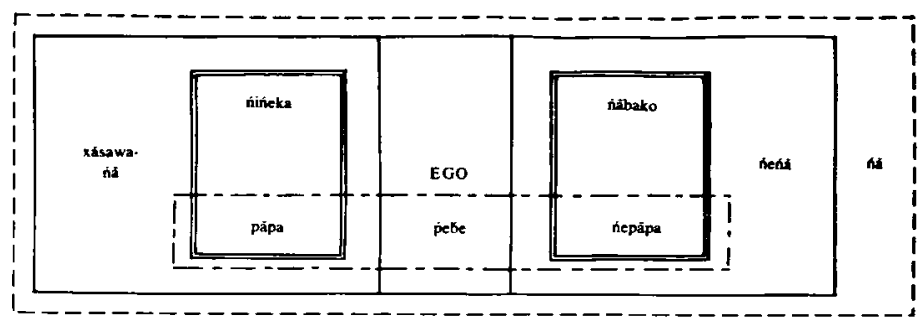

Abb. ?

Die Bedeutung des reinen $\dot{n} \bar{a}$ ist wesentlich umfassender als die der Termini xāsawańā oder ńeńa, d.h. in diesem Fall wird weder nach Genus noch nach Alter differenziert; die Benennung bezieht sich also auf jedes Mitglied der mit EGo auf derselben Generationsebene liegenden Seitenlinien-Verwandtschaft, wenn es sich um dieselben Eltern handelt. Es ist leicht einzusehen, dass sich die unterscheidenden Elemente $x \bar{a} s a u a$ und ne wie Präfixe verhalten. Es ist eine systemtheoretische Tatsache, dass in einer Menge mit nur zwei Elementen lediglich das eine mit einem differenzierenden Element versehen zu werden braucht. Aus bereits oben dargelegten Gründen ist es nahezu natürlich, dass das unbezeichnete Glied auf den Mann hinweist, das bezeichnete (also: ne-ña) auf die Frau. Die Herausstellung des Elements xäsaua ist möglich, aber nicht bindend.

Oben wurde bereits darauf hingewiesen, dass die Verwandtschaftstermini der Nenzen weitgehend polysem sind. Das lässt 
$+2$

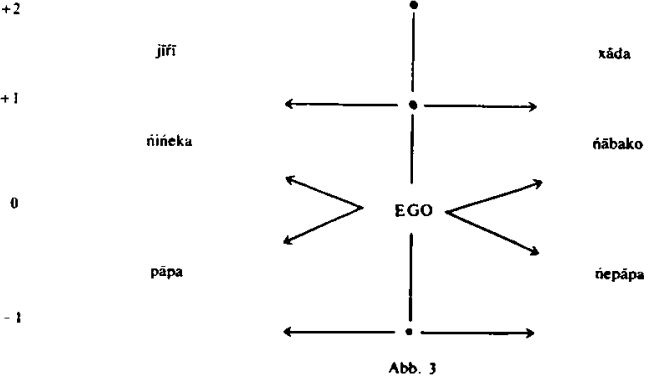

sich auch durch die Beispiele aus dem Bereich der SeitenlinienVerwandtschaft untermauern.

Mit jḯr werden all jene männlichen Verwandten bezeichnet, die mehr als eine Generation älter sind als EGo. Dieser Terminus folgt der Gentillinie nicht streng, denn es gibt sowohl auf väterlicher als auch auf mütterlicher Seite gleichermassen Verwandtschaftsverbindungen vom Typ jirí. Das gilt auch für die Bezeichnung $x \bar{a} d a$.

Mit nińelia werden männliche Angehörige bezeichnet, die älter sind als EGo, aber jünger als der Vater. Diese Verwandten gehören regelmässig zur Verwandtschaft väterlicherseits. Die symmetrische weibliche Entsprechung lautet náabako. Das genaue Spiegelbild auf der absteigenden Linie bilden pāpa und riepāpa.

Die bisherigen Ausführungen zeigen, dass die einzelnen Termini -- je weiter wir uns hinsichtlich des Lebensalters von EGo entfernen - immer mehr Bedeutungselemente enthalten: davon hat der dem Ego am nächsten stehende Terminus pebe am wenigsten und das Paar jīri $-x \bar{a} d a$ am meisten.

5.3. Auf entferntere Positionen in der Seitenlinien-Verwandtschaft weisen $\dot{m} \bar{a} \eta$ 'Sohn der Schwester, Sohn der Schwester des Vaters' und nemā̄ 'Tochter der Schwester, Tochter der Schwester des Vaters'.

Von einigen besonderen Ausdrücken für Verbindungen mütterlicherseits soll eigens die Rede sein. Bei der Spezifikation dieser Verwandtschaftsbeziehungen kann es sich nicht um einen blossen Zufall handeln; es scheint, als habe sich in den Namen die Spur einer solchen Kette von verwandtschaftlichen Verbindungen erhalten, die einmal sehr wichtig waren. Es ist 
letzten Endes anzunehmen, dass der Ursprung dieser sprachlichen Belege irgendwo im Matriarchat zu suchen ist. Hierher gehören die Termini tidā 'jüngerer Bruder der Mutter, Sohn des Bruders der Mutter; Sohn der jüngeren Schwester des Vaters' und neje 'jüngere Schwester der Mutter, Tochter des Bruders der Mutter; Tochter der Schwester des Vaters'. Beide sind i.a. älter als Eqo. Dass auch die väterliche Linie unter diesen Bedeutungen vertreten ist, weist auf eine Art Bedeutungserweiterung hin. Sicher ist, dass diese Bedeutungen relativ jungen Ursprungs sind.

5.4. Bei der Betrachtung jener verwandtschaftlichen Beziehungen, die durch die Eheschliessung zustande kommen, ist das Genus von EGo nicht mehr nebensächlich. So beziehen sich die Bezeichnungen denn auch meist auf kleinere oder grössere Gruppen. Die Unterscheidung der Mutter mittels einem bezeichnenden Terminus ist gesellschaftlich notwendig, denn die Verbindung zwischen EGo und der Mutter ist auch selbst von unterscheidender Bedeutung. Ansonsten stammt die Mutter - als Ehefrau des Vaters - ja aus einer anderen Gens, wie es die Regeln der Exogamie vorschreiben.

Von jedem Verwandten namens ńinela heisst die Ehefrau ńäba, die maximal eine Generation älter ist als EGo.

Die durch Eheschliessung entstehenden verwandtschaftlichen Beziehungen vermögen zu erhellen, wie aus einer ñäba z.B. die Stiefmutter von EGo werden kann. Die »Beförderung» zur Stiefmutter bedeutet bei den Nenzen keinen solchen Bruch, wie das Wort unseren Begriffen nach eingibt. Die $\dot{n} \bar{a} b a$ ist bereits früher ein Mitglied der Familie, d.h. mit Ego verwandt. So ändert sich höchstens ihr wrechtlicher Status» in der Familie.

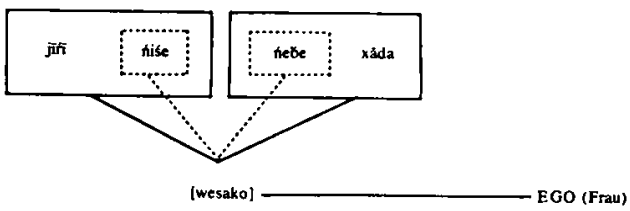


Häufig wird der obige Ausdruck durch das Wort nebte ersetzt, wenn nämlich die betreffende jünger ist als die Mutter von EGo.

Wie die obigen Beispiele zeigen, beträgt der Altersunterschied zwischen EGo und jiŕri oder $x \bar{a} d a$ wenigstens zwei Generationen. Wenn aber Ego eine Frau ist, zeigt sich die $\bar{j} \bar{\imath} \hat{r} \bar{\imath}-x \bar{a} d a$-Verwandtschaft schon in der ersten Generation. Diese Annäherung im Altersunterschied lässt sich nur so lösen, wenn wir berücksichtigen, dass Mann und Frau jeweils zu einer anderen Gens gehören. Jene gensgebundene Differenz entspricht in dem gegebenen Fall der Generationsstufung innerhalb einer Gens.

Im Zusammenhang mit der Eheschliessung werden mitunter erstaunlich ferne Verhältnisse benannt. I.a. lehren die Beispiele, dass die von EGo aus gesehen um eine (oder mehr) Generationen älteren Verwandten eigentlich nicht spezifiziert werden, wenn diese Verwandtschaft zur Gens des Ehepartners gehört. Ent-

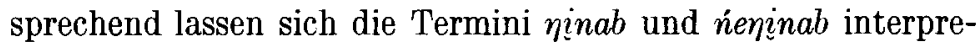
tieren. Verglichen mit dieser hochgradigen Verallgemeinerung treffen wir bei den Verwandtschaftsbeziehungen innerhalb einer Generation auf eine verblüffende Spezifizierung (natürlich auch weiterhin im Kreise der klassifizierenden Termini). Eine engere Verbindung wird ausgedrückt durch nädo 'jüngerer Bruder der Frau, jüngerer Bruder des Mannes' und nenādo 'jüngere Schwester der Frau, jüngere Schwester des Mannes' oder ninedi 'Mann der Schwester'. Im Falle von śel 'Mann einer Schwester des Mannes, Mann einer Schwester der Frau', nesel 'Frau eines Bruders der Ehefrau, Frau eines Bruders des Mannes' oder bei dem Paar jāne? und ńejāne? beispielsweise liegen verwickeltere Zusammenhänge vor.

Für die Verwandtschaftsbeziehungen ist der Tod das, was in der Musik das Auflösungszeichen bedeutet. Es hängt wahrscheinlich mit dem Phänomen des Tabu zusammen, dass es kaum sprachliche Dokumente gibt für diese wichtigen Umstände. Hierher gehört z.B. auch der Terminus jewako 'Waise', der sich nur auf den Fall bezieht, dass die Eltern sterben. Stirbt das Kind, bleibt diese Tatsache ohne sprachliche Benennung. Die die Auflösung der Ehe ausdrückenden Termini sēra (xäsawa) und sēra ne werden wohl sehr jungen Ursprungs sein.

6. Bereits einleitend wurde erwähnt, dass bei den samoje- 
dischen Sprachen oft sehr wichtige Angaben fehlen. Diese Feststellung gilt ganz besonders für das Selkupische. Aus beiden von mir untersuchten Dialekten fehlen uns solche Angaben, die von Fall zu Fall vielleicht entscheidend sein könnten. ${ }^{1}$

6.1. Das System der Verwandtschaftsnamen der direkten Linie hat sich in den beiden Dialekten anders entwickelt.

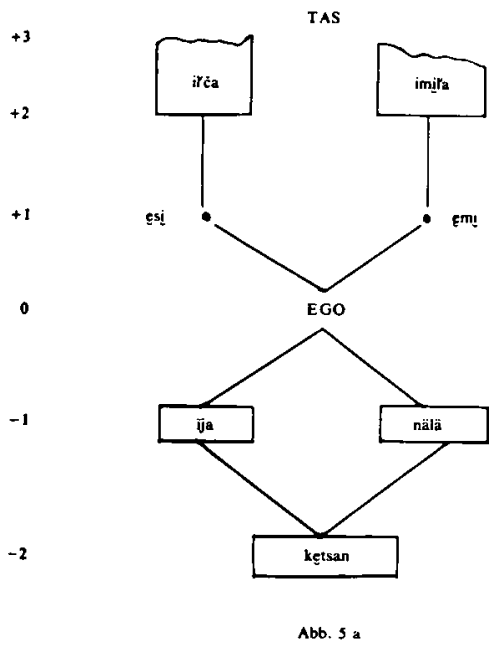

Im Tas-Dialekt begegnen wir häufig auch dann einem klassifizierenden Terminus, wenn im Narym ein bezeichnender Ausdruck vorliegt (z.B. auf der 2. Stufe der aufsteigenden Linie). Auch auf der absteigenden Linie ist die Narym-Mundart analysierender, d.h. bei den Enkeln wird auch hinsichtlich des Genus unterschieden. Tas $i j a$ und Narym $j i$ sind gleichermassen anwendbar in der Bedeutung von 'Sohn' und 'Kind'. Über die Erklärung war bereits im Zusammenhang mit dem Nenzischen die Rede.

Neben den mehr analysierenden Formen im Narym haben sich auch Spuren solcher Termini erhalten, die eine umfassendere Bedeutung haben, d.h. sich auch auf mehrere Verwandten

${ }^{1}$ Als Quelle wurden die folgenden Werke herangezogen: Castrén Lehtisalo, MSFOu 122; Pápai - Hajdú, NyK 54 141-84; István Erdélyi, Selkupisches Wörterverzeichnis. Budapest 1969. 
beziehen können. So existiert z.B. palda 'Mutter meines Vaters, Mutter meiner Mutter' unabhängig davon, dass es auch getrennt eine Bezeichnung adva und aunemba gibt.

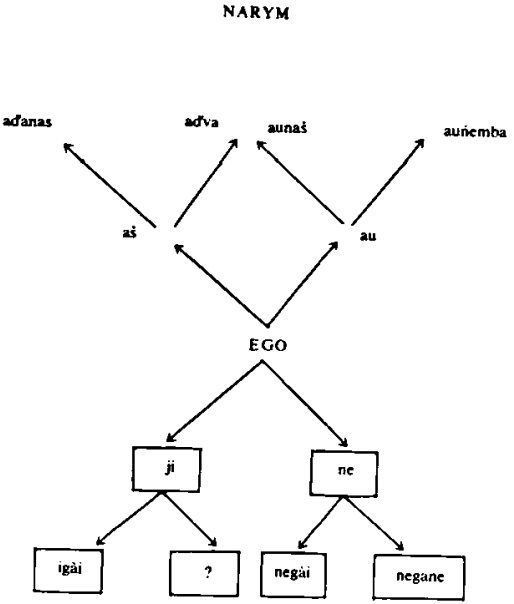

Abb. 5 b

6.2. Bei der Seitenlinien-Verwandtschaft ist im vorliegenden Fall ebenfalls die Berücksichtigung der von identischen Eltern abstammenden Geschwister am wichtigsten.

Die Tas-Formen können den relativen Altersunterschied nur durch Umschreibung ausdrücken, während es im Narym einen Terminustyp gibt, der nur aufgrund eines relevanten Merkmals (nach dem Genus) klassifiziert: tebena und neria; es existiert jedoch noch ein anderer Typus, wo man mit zwei unterscheidenden Merkmalen arbeiten muss (mit dem Genus und dem Altersunterschied zu EGo).

Die Verwandtschaft auf der Seitenlinie breitet sich natürlich in weitere Richtungen aus. Sprachlich können wir das jedoch nur erahnen. Wir verfügen über insgesamt zwei wirklich sichere Angaben, die sich auf diese verwandtschaftlichen Verbindungen beziehen: in der aufsteigenden Linie ojna 'jüngere Schwester der Mutter' und in der absteigenden Linie keča 'Sohn der Schwester'. 

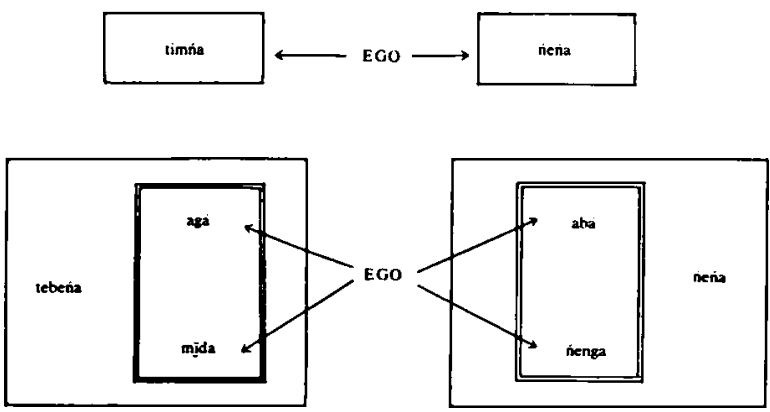

Abb. 6

Im Narỵm-Dialekt ist die Situation nicht viel günstiger. Aller Wahrscheinlichkeit nach bezieht sich ilda auf männliche Verwandte, die eine Generation älter sind als EGo, die Brüder des Vaters oder der Mutter sind. Wir müssen annehmen, dass es einen entsprechenden Terminus auch für die weiblichen Verwandten geben kann. Auf der absteigenden Linie bedeutet ńeńna die Töchter der Geschwister von EGo, während sich hier für die Söhne bereits zwei Termini anbieten (köba ilda, kad'a). Für einen genauen U̇berblick über das System reichen also die Angaben keinesfalls aus.

6.3. Durch die Ehe wird die Verwandtschaft der Seitenlinie weiter ausgebaut. Für die Benennung dieser Relationen werden jedoch eine Reihe von Termini verwendet, die wir bereits von der Blutsverwandtschaft her kennen.

Die Ehepartner werden in der Tas-Mundart durch qup und ima wiedergegeben, im Narym durch téde und ema.

Für die Bezeichnung der Eltern des Ehepartners ist das Genus von EGo völlig nebensächlich. Narym ilda bezieht sich gleichermassen auf den Vater des Jannes und der Ehefrau; so verhält sich auch die Tas-Entsprechung (qen $\underline{i}$ ira). Ähnlich unwichtig ist das Genus von EGo in der absteigenden Linie, d.h. die Bedeutung 'Schwiegertochter' und 'Schwiegersohn' für Narym $\dot{e} m n \dot{e}$ und indima, unabhängig davon, ob EGo ein Mann oder eine Frau ist.

An den weiter entfernt liegenden Punkten der nicht-natürlichen Verwandtschaft begegnen Termini, die wir bereits von 
früher her kennen. Tas ōna beispielsweise bedeutet in der Blutsverwandtschaft in der Seitenlinie die 'jüngere Schwester der Mutter', in unserem Zusammenhang hier jedoch 'Ehefrau meines älteren Bruders'. Die semantische Struktur dieses Terminus sollte vielleicht näher betrachtet werden. Bei der Relation 'jüngere Schwester der Mutter' spielen u.a. die folgenden Bedeutungselemente eine Rolle: weibliche Verwandte; nicht in direkter Linie verwandt; Altersunterschied tritt in den Hintergrund. Bei der Verwandtschaftsbeziehung 'Frau meines älteren Bruders' findet sich folgendes: weibliche Verwandte; nicht in direkter Linie verwandt, nur infolge von Heirat; Altersunterschied tritt in den Hintergrund, die betreffende Person kann älter und jünger als EGo sein. Mit einer ähnlichen Erscheinung haben wir es im Falle von Narym ilda zu tun, welcher Terminus bekanntlich sowohl für die Blutsverwandtschaft als auch für die nichtnatürliche Verwandtschaft verwendet wird. Natürlich ist die Bedeutungsstruktur der Termini in beiden Fällen viel komplizierter, doch wird gerade durch die Bestimmung der wichtigsten relevanten Merkmale klar, wie es möglich ist, dass für derart weit voneinander entfernte verwandtschaftliche Positionen ein und dieselbe Benennung verwendet wird.

Es gibt innerhalb der Verwandtschaftsterminologie einige ziemlich vernachlässigte Gebiete. Hierher gehört u.a. auch die Verbindung zwischen den Eltern der Ehepartner. Im NarymDialekt wird siadibaja 'Mitmutter, Schwippschwägerin, Mutter des Ehepartners des Kindes' verwendet und svadiéra 'Schwippschwager, Vater des Ehepartners des Kindes'. Die Vorsilbe der Termini fungiert offenbar als Präfix: es wird dadurch jene nichtnatürliche Verwandtschaft ausgedrückt, die durch die Heirat der Kinder der diesbezüglichen Personen zustande kommt.

7. Eine zusammenfassende Betrachtung der Verwandtschaftshierarchie im Samojedischen ist nicht möglich. Dazu wäre ein wesentlich vollständigeres Material notwendig. Die Wörterbücher bringen mitunter eine grössere Zahl von Termini als oben angeführt, doch können sie doch nicht herangezogen werden, da sie schlecht oder mangelhaft definiert sind. Vor allem das selkupische, aber auch das nenzische Material müsste 
durch neue Sammlungen ergänzt werden, durch die viele sprachlichen, ethnologischen u.a. Fragen in ein neues Licht rücken würden.

Aus dem gleichen Grund ist es nicht möglich, einen sehr charakteristischen Zweig der Verwandtschaftsterminologie zu behandeln, nämlich die Anredeformen. Es ist allgemein bekannt, dass häufig ein anderer Terminus verwendet wird, wenn wir den betreffenden Verwandten selbst anreden und dabei auf die verwandtschaftliche Beziehung zwischen EGo und der angeredeten Person hinweisen. Es ist möglich, aber durchaus nicht gesetzmässig, dass die Anrede mit dem bezugnehmenden Terminus zusammenfällt (wenn man z.B. zu einem Dritten etwas über die in Frage stehende verwandte Person sagt).

Den Anredeterminus finden wir auch im Nenzischen: abalulu 'Grossmutter', mamako 'Mutter', desgleichen im Selkupischen: mama 'Mutter', apa 'Vater', ölga 'so redet die Ehefrau die Schwester des Mannes an'. Die meisten Belege entstammen der Kindersprache. Onomatopoetische Wörter treten häufig in recht weit voneinander entfernten Sprachen in ähnlicher Lautgestalt auf. Setälä meinte von den östlichen Sprachverwandten der Finnen, bei ihnen sei das System der Anredetermini überhaupt nicht ursprünglich, sondern es habe sich völlig unter dem Einfluss der Kultur türksprachiger Völker entwickelt. Dem kann man natürlich in dieser Form nicht ganz zustimmen, während ein bestimmter Einfluss mit Sicherheit als Tatsache anzuerkennen ist.

Es hat den Anschein, als habe sich das ursprüngliche System der verwandtschaftlichen Bezeichnungen im Nenzischen erhalten, und als verweise das Selkupische bereits auf einen etwas neueren gesellschaftlichen Zustand. Rückschlüsse auf die gesellschaftliche Hierarchie sind jedoch stets als vorsichtige Vermutungen zu sehen, denn wir haben das Beispiel der beiden Selkup-Mundarten deutlich vor Augen, wo sich auf einer relativ identischen Basis voneinander stark abweichende Systeme entwickelten.

TAMÁS MÁRK 